\title{
Completely Pinpointing the Missing RFID Tags in a Time-efficient Way
}

\author{
Xiulong Liu, Keqiu Li*, Geyong Min, Yanming Shen, Alex X. Liu, Wenyu Qu
}

\begin{abstract}
Radio Frequency Identification (RFID) technology has been widely used in inventory management in many scenarios, e.g., warehouses, retail stores, hospitals, etc. This paper investigates a challenging problem of complete identification of missing tags in large-scale RFID systems. Although this problem has attracted extensive attention from academy and industry, the existing work can hardly satisfy the stringent real-time requirements. In this paper, a Slot Filter-based Missing Tag Identification (SFMTI) protocol is proposed to reconcile some expected collision slots into singleton slots and filter out the expected empty slots as well as the unreconcilable collision slots, thereby achieving the improved time-efficiency. The theoretical analysis is conducted to minimize the execution time of the proposed SFMTI. We then propose a cost-effective method to extend SFMTI to the multireader scenarios. The extensive simulation experiments and performance results demonstrate that the proposed SFMTI protocol outperforms the most promising Iterative ID-free Protocol (IIP) by reducing nearly $45 \%$ of the required execution time, and is just within a factor of 1.18 from the lower bound of the minimum execution time.
\end{abstract}

Index Terms—RFID systems, Missing Tag, Completely Pinpointing, Time Efficiency, Optimization.

\section{INTRODUCTION}

C OMPARED to the traditional barcode technology, the newly emerging Radio Frequency Identification (RFID) technology possesses many attractive advantages, e.g., (1) line of sight is not required for reading; (2) multiple items can be read with a single scan; and (3) tags can be read from a relatively long distance. As a result, RFID systems are being increasingly used in various applications such as localization [1], [2], [3], supply chain management [4], [5], [6], warehouse management [7], [8], [9], etc. An RFID system usually consists of RFID readers and a large number of tags. RFID tags are labeled in designated objects where each tag has a small size of memory to store its unique ID and some other information (e.g., product price, expiry date, personal information, etc). There are two types of tags [10]: (1) passive tags that are powered up by harvesting the radio frequency energy from readers and have communication range often less than 20 feet; (2) active tags that have their own power sources and have relatively longer communication ranges. A reader has a dedicated power source with significant computing power. It transmits a query to a set of tags, and the tags respond over a shared wireless medium.

- X. Liu, K. Li and Y. Shen are with the School of Computer Science and Technology, Dalian University of Technology, No 2, Linggong Road, Dalian 116023, China.E-mail: keqiu@dlut.edu.cn.

- G. Min is with the Department of Computing, University of Bradford, Bradford, BD7 1DP, United Kingdom. E-mail: G.Min@brad.ac.uk.

- Alex X. Liu is with the Department of Computer Science and Engineering, Michigan State University, East Lansing, MI, U.S.A.. E-mail: alexliu@cse.msu.edu.

- W. Qu is with the School of Information and Technology, Dalian Maritime University, Dalian 116026, China. E-mail: eunice.qu@gmail.com.
This study investigates the challenging problem of complete identification of missing tags in largescale RFID systems. For example, imagine a large warehouse with tens of thousands of items, such as refrigerators, televisions and bicycles. One of the most fundamental tasks is to monitor whether some items are missing due to management fault or theft. A traditional method is to manually check them one by one, which suffers two significant drawbacks. (1) Poor accuracy: a thief may deliberately steal some blocked items to conceal this theft. Then, the absence of these missing items is difficult to discover; (2) Long checking interval: obviously, this manual checking process is seriously laborious and thus cannot be conducted continuously. The interval between any two checking processes is usually long enough for the thief to escape. As an emerging technology, RFID could be used in the above monitoring application (if an RFID tag is missing, the corresponding tagged item is also treated as missing) to tackle these drawbacks of the manual checking method. Since RFID is a wireless communication technology, it does not require the line of sight. The presence of the tagged items can be accurately checked even if they are blocked behind other items. Moreover, this RFID-based monitoring processes can be performed continuously, and the the interval of any two consecutive checking processes can be as short as possible. As a result, the theft event can be discovered in time. Similar applications also exist in other scenarios, e.g., retail stores, hospitals, prisons, etc.

Although this problem has attracted extensive attention from academy and industry, the existing work still can hardly satisfy the stringent real-time requirements. To the best of our knowledge, a promising 
missing tag identification scheme is the Iterative IDfree Protocol (IIP) proposed in [8]. IIP is a variant of the Framed Slotted Aloha protocol where all the tags use their IDs and a hash function to pseudo-randomly pick a slot in the time frame so as to respond and announce their presence. Because the reader knows all the tag IDs as well as the used hash function, it is able to predict the empty slot that is expected to contain no tag response; the singleton slot that is expected to contain one and only one tag response; the collision slot that is expected to contain two or more tag responses. In fact, some of the tags may be missing, which makes it possible that the actual status of a slot different from its expected status. By comparing the observed slot statuses with the expected statuses, the reader can identify the missing tags. Specifically, if an expected singleton slot turns out to be an empty one, the reader asserts that the tag corresponding to this slot is missing. However, the expected empty slots and the expected collision slots are not used and wasted, which decreases the efficiency of IIP. Although the authors investigated a method [8] to turn some of the expected collision slots into the expected singleton slots in order to improve the efficiency of IIP, the effect of collision reconcilement is limited (only a small fraction of the expected collision slots could be reconciled into the singleton ones). Moreover, the deficiency due to the expected empty slots are not noticed and still wasted directly. According to the theoretical analysis in [8], we find that the expected empty slots and expected collision slots still account for nearly $48 \%$. That is, IIP is of low time-efficiency and has a large room to be improved.

To approach a real-time missing tag identification, this paper proposes a Slot Filter-based Missing Tag Identification (SFMTI) protocol, which possesses two significant advantages over IIP. (1) a more effective collision reconcilement method is investigated, which can turn more collision slots into the expected singleton ones; (2) the expected empty slots and the unreconcilable collision slots are filtered out and not executed in order to improve time-efficiency. In the proposed SFMTI protocol, all the executed slots are expected to be singleton, and thus each of them is useful for the missing tag identification. The above two advantages precisely relieve the drawbacks of IIP. This paper theoretically analyzes the parameter settings to minimize the execution time of the proposed SFMTI. The extensive simulation results demonstrate that this new SFMTI protocol outperforms the most promising IIP scheme by reducing $45 \%$ of the required execution time.

The major contributions of this paper are summarized as follows:

1) A new SFMTI protocol is proposed to efficiently identify the missing tags, by reconciling collision slots into the expected singleton slots and filter- ing out the expected empty slots as well as the unreconcilable collision slots.

2) This paper theoretically analyzes the performance of the proposed protocol and optimizes the parameter settings to achieve the best timeefficiency.

3) A cost-effective method is proposed to extend the SFMTI protocol to the multi-reader scenarios, where the readers can perform the missing tag identification in a parallel mode.

4) Extensive simulation experiments are conducted to evaluate the performance of the proposed protocol. The simulation results match well with the analytical results and demonstrate the proposed protocol performs much better in terms of execution time than the currently most promising protocol.

The rest of this paper is organized as follows. Section 2 surveys the related work. Section 3 presents the system model and problem description. The detailed SFMTI protocol is presented in Section 4. Section 5 evaluates the performance of the SFMTI protocol. Finally, Section 6 concludes this paper.

\section{Related Work}

In its infancy stage of the RFID technology, the tag collection problem, which is to collect the IDs from a large number of tags as quickly as possible, attracted extensive research attention. The solutions to tag collection problem are generally classified into two categories: Aloha-based protocols [11], [12], [13] and Tree-based protocols [14], [15], [16].

The former works as follows. The reader first tells the frame size $f$ and a random number $R$ to the tags in its vicinity. Each tag then uses the received parameters $f, R$ and its ID to select a slot in the frame by calculating a hash function $h(I D, R) \bmod f$ whose result is in $[0, f-1]$ following a uniform distribution. Then each tag responds its ID in the selected slot. In any slot, if one and only one tag responds, the reader is able to successfully get the ID information of that tag. This type of slot is referred to as a singleton slot. An RFID tag that is successfully collected in a singleton slot will keep silent for the rest of the collection process. If multiple tags simultaneously transmit their IDs in a common collision slot, the responses are garbled due to collision and thus retransmission is required. The collection process does not terminate until all the tags are collected [10].

On the other hand, a Tree-based protocol [14], [15], [16], [17] organizes all IDs in a binary tree where the height of this tree is equal to the length of a tag ID. Each left branch of the tree is marked by ' 0 ' and each right branch by ' 1 '. As a result, an arbitrary tag ID corresponds to a unique path from the root to a leaf. A reader first queries ' 0 ' and all the tags whose IDs start with ' 0 ' respond. If the result query is a successful 
read (i.e., exactly one tag responds) or an empty read (i.e., no tag responds), the reader queries ' 1 ' and all the tags whose IDs start with ' 1 ' respond. If the result of the query is a collision, the reader generates two new query strings by appending a ' 0 ' and a ' 1 ' to the previous query string and queries the tags with these new query strings. All the tags whose IDs start with the new query string respond. This process continues until all the tags have been identified [17].

In recent years, RFID technology is widely used for many monitoring applications, where the missing tag problem is yet under-investigated in the research community. The missing tag problem can be generally classified into two categories: (1) the missing tag detection focuses on detecting whether any RFID tags are missing instead of exactly identifying which ones are missing. (2) the missing tag identification concentrates on identifying the exact missing tags.

Obviously, the solutions to tag collection problem can solve the missing tag problem by collecting all the tag IDs and then comparing the collected IDs with the ID information stored in the database. However, these methods are seriously time-consuming because of recollecting a large number of reductant IDs.

In order to efficiently address the problem of missing tag detection, Tan et al. proposed the Trust Reader Protocol (TRP) to detect the missing-tag event with a predefined probability $\alpha$ when the number of the missing tags exceeds $m$ (a tolerance threshold) [18]. To improve the time-efficiency and energy-efficiency of TRP, Luo et al. introduced the sampling idea, and thus proposed the Efficient Missing-tag Detection (EMD) protocol, where the detection result on the sampled tags is used to probabilistically reflect the whole intactness of RFID systems [19]. Based on their prior work, Luo et al. proposed a multi-hash approach to further improve the performance of the missing tag detection protocol in [20]. The above schemes can only detect the missing tag event but cannot exactly find out which tags are missing and thus fail to provide the details of the missing tags.

The problem of missing tag identification is also of great practical importance, and many efforts have been made to address it. Unfortunately, the existing solutions can hardly satisfy the stringent real-time requirements. The most promising missing tag identification protocol is the Iterative ID-free Protocol (IIP) proposed in [8]. Its basic principle is described in Section I, and its deficiency is also analyzed. In [21], Zhang et al. investigated the problem of missing tag identification in the multi-reader scenarios, where all the readers perform synchronized and parallel scans. The authors claimed that their best protocol (i.e., Protocol 3) reduces the time for identifying all the missing tags by up to $75 \%$ in comparison to IIP. In fact, the superiority of their protocol over IIP benefits from the cooperation of the readers. In the single reader scenarios (or in the scenarios where the number of readers is small), IIP still runs faster than the Protocol 3 in [21].

\section{System Model and Problem De- SCRIPTION}

\subsection{System Model}

We consider a large RFID system with a single reader and $N$ tags where all the tags are within the interrogating range of this reader. Please note that, for the purpose of clarity, we first present the SFMTI protocol in the case of a single reader and then extend the use of this protocol in large-scale RFID systems with multiple readers. The tag set is denoted as $S_{\text {all }}$, i.e., $S_{\text {all }}=\left\{t_{1}, t_{2}, \ldots, t_{i}, \ldots, t_{N}\right\}$. Each tag, say $t_{i}$, has a unique $I D_{i}$ and is equipped with the same uniform Hash generator $H(\cdot)$. The reader has access to a database that stores the IDs of all tags. The reader communicates with the central computer through a high-speed network link.

\subsection{Communication Overview}

The reader continuously sends synchronization signals to create a slotted time frame. The interactive communications are in the Reader Talks First (RTF) mode [22], i.e., the reader queries the tags first, and a tag picks a slot to respond according to the reader's commands. Li et al. classified the slots into three categories: tag slots, long-response slots and short-response slots, based on their length [8]. The length of a tag slot is denoted as $t_{\text {tag }}$, which allows the transmission of a tag ID (96 bits), either from the reader to the tags or from a tag to the reader. The length of a longresponse slot is denoted as $t_{\text {long }}$, which can afford transmitting a long response carrying 10 bits information. The length of a short-response slot is denoted as $t_{\text {short }}$, which allows the transmission of a shortresponse carrying only one bit information. Based on the specification of the Philips I-Code system [23], the length $t_{\text {tag }}$ of tag slot is set to $2.4 \mathrm{~ms}$ (including the wait time between any two consecutive transmissions) for transmission of a tag ID (96 bits) from a tag to a reader or vice versa; the length $t_{\text {long }}$ and $t_{\text {short }}$ are set to $0.8 \mathrm{~ms}$ and $0.4 \mathrm{~ms}$, respectively [8].

\subsection{Problem Statement}

Some tags may be missing due to theft, management fault, etc. We do not know which tags are missing and even the number of the missing ones. The problem addressed in this paper is to quickly identify all the missing tags. Obviously, the execution time is the most important performance metric for a missing tag identification protocol. The used notations are summarized in Table 1. 
TABLE 1

Notation used in this paper.

\begin{tabular}{|c|l|}
\hline Symbol & Description \\
\hline$N$ & The number of tags in the system \\
\hline$S_{\text {all }}$ & The set of all tags in the system \\
\hline$S_{\text {miss }}$ & The set of the missing tags \\
\hline$I D_{i}$ & The ID of Tag $t_{i}$ \\
\hline$N^{*}$ & The number of the tags participating in the current round \\
\hline$f$ & The length of the filter vector \\
\hline$\rho$ & A variable given by $N^{*} / f$ \\
\hline$R$ & The random number that is fresh in each round \\
\hline$H(\cdot)$ & The Hash generator with a uniform random distribution \\
\hline$e$ & $\begin{array}{l}\text { The natural constant which is approximately equal to } \\
2.71828\end{array}$ \\
\hline$C$ & The expected execution round count \\
\hline
\end{tabular}

\section{The Proposed Slot Filter-based Missing TAG IDENTIFICATION PROTOCOL}

This section will first analyze the deficiency of two typical solutions for identifying the missing tags, which inspires the motivation and ideas of the proposed SFMTI protocol. We will then present the SFMTI protocol in detail and investigate how to optimize its parameter settings. After that we propose a cost-effective method to extend the proposed SFMTI protocol to the multi-reader scenarios.

\subsection{Motivation}

\subsubsection{An Ideal Lower Bound}

A widely accepted lower bound on the minimum execution time for the problem of missing tag identification was given in [8], which is redescribed as follows. All $N$ tags relay 1-bit short-responses one after another to declare their presence. If the reader does not receive a response as expected, the corresponding tag must be missing. Clearly, one scanning is adequate to verify the presence of all $N$ tags. If the transmission of control information is not considered, the execution time is $N \times t_{\text {short }}$, where $t_{\text {short }}$ is the time for transmitting a 1-bit response. Although this lower bound is unlikely to be achieved because the reader has to transmit control information to coordinate the protocol execution, it offers a guidance and also a target for any missing tag identification protocols to achieve.

\subsubsection{A Straightforward Polling Protocol}

The most straightforward way for identifying the missing RFID tags is a polling method [8]. As the reader gets full knowledge of all the tag IDs stored in a database, it could request the IDs one by one. A tag responds a short-response if it finds that the current request contains its ID information. Clearly, if the reader receives a response as expected, the requested tag must be present; otherwise, this tag is missing. The execution time of this polling method is $N \times\left(t_{\text {tag }}+t_{\text {short }}\right)$, where $t_{\text {tag }}$ is the time for transmitting a tag ID. This polling method is far from the the lower bound, and the key reason is that transmission of IDs is extremely time-consuming.

\subsubsection{The Most Promising Protocol}

The Iterative ID-free Protocol (IIP) [8] is a promising missing tag identification protocol which is a variant of the classical Framed Slotted Aloha protocol. IIP avoids the transmission of IDs, which uses the expected singleton slots to verify the presence of the corresponding tags. Specially, if an expected singleton slot turns out to be empty, the corresponding tag must be missing.

However, according to the theoretical analysis in [8], we find that the expected empty slots and the expected collision slots in IIP account for nearly $48 \%$, which degrades its efficiency. Clearly, IIP still has a large room to be improved. Comparing the performance results of IIP with the ideal lower bound, the gap between them is that IIP contains a large proportion of expected empty slots and collision slots. Hence, the key to approaching the ideal lower bound is how to reduce (or eliminate) the expected empty slots and collision slots, which is a challenging issue.

\subsection{Protocol Design}

The proposed Slot Filter-based Missing Tag Identification (SFMTI) protocol possesses two main innovation points: (1) reconciling some expected collision slots into the expected singleton slots; (2) avoiding the execution of the expected empty slots and the unreconcilable collision slots, which precisely relieves the drawbacks of IIP. The proposed SFMTI includes multiple rounds, each of them consists of three stages: (1) Collision Slot Reconciling stage, in which the reader reconciles the expected collision slots and turns some of them into the expected singleton ones; (2) Slot Filtering stage, in which the reader creates a filter vector to filter out the expected empty slots and the unreconcilable collision slots. (3) Presence Verifying stage, in which the tags that pick the expected singleton slots respond shot-responses to announce their presence. In a round, the presence of some tags are able to be verified. SFMTI repeats for multiple rounds until all the tags are verified. In what follows, we present this protocol in detail.

\subsubsection{Collision Slot Reconciling Stage}

As aforementioned, the reader gets full knowledge of the tag IDs. Then, it uses a uniform hashing function $H(\cdot)$ and a random number $R_{1}$ to map all the IDs to a filter vector containing $f$ elements, each of which corresponds to a slot. For example, a tag $t_{i}$ is mapped to the $s_{i}^{t h}$ element of the filter, where $s_{i}=H\left(I D_{i}, R_{1}\right)$ $\bmod f$. As a result, there are generally three types of slots: the expected empty slot, to which no tag is mapped; the expected singleton slot, to which one and only one tag is mapped; the expected collision slot, to 


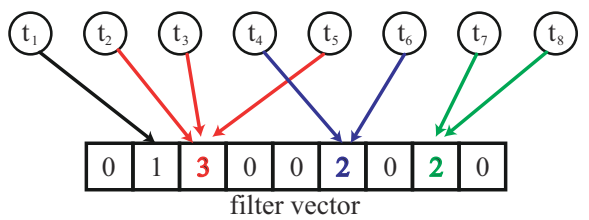

(a)

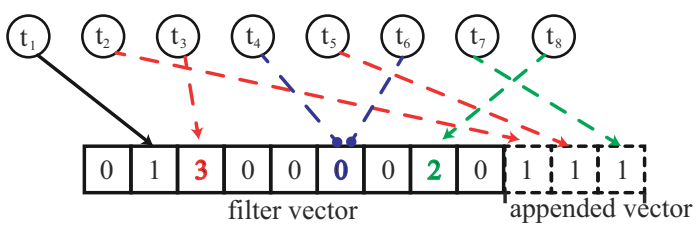

(b)

Fig. 1. An example of reconcilement of $k$-collision slots $(k=2$ or 3$)$. (a) Mapping all the tags to a filter vector with $f$ elements; (b) Relocating the tags confined in the reconcilable collision slots.

which two or more tags are mapped. To mark these 3 types of slots, the length $\ell$ of each element in the filter is at least 2 bits. For the clarity of description, we assume $\ell=2$, i.e., each element in the filter vector has 2 bits. Then the elements corresponding to the expected empty slots are set to ' $0 s^{\prime}$; the elements corresponding to the expected singleton slots are set to ' $1 \mathrm{~s}$ '. Let $k$-collision slot denote the slot to which $k$ tags are mapped at the same time. The elements corresponding to the 2-collision slots and 3 -collision slots are set to ' $2 \mathrm{~s}$ ' and ' $3 \mathrm{~s}^{\prime}$, respectively. Intuitively, the larger the $k$ is, the harder the collision reconcilement is. Moreover, to distinguish more types of collision slots (e.g., 4-collision slots, 5-collision slots, etc.), 2 bits per element are not enough, and we have to use 3 or more bits per element to mark these status of slots. As a result, the overhead of transmitting the filter vector increases $50 \%$ when each element is 3 bits. Hence, we do not try to reconcile the $k$-collision slots $(k \geq 4)$, and the elements corresponding to these collision slots are directly set to ' $0 s^{\prime}$. The above process is exemplified in Fig. 1 (a).

The reader generates another random number $R_{2}$ to reconcile the 2-collision slots and 3-collision slots. What is the collision reconcilement? For a certain $k$ collision slot $(k=2$ or 3$)$, the reader uses the new random number $R_{2}$ to calculate $H\left(I D, R_{2}\right) \bmod k$ for each tag confined in this slot, the hashing result is within $[0, k-1]$. If each of the $k$ tags is hashed to a unique number $b \in[0, k-1]$, we say this $k$-collision slot is successfully reconciled; otherwise, this slot is unreconcilable. This collision reconciling process is exemplified in Fig. 1 (b). For example, tags $t_{2}, t_{3}$ and $t_{5}$ are mapped to a 3 -collision slot in Fig. 1 (a). By performing $H\left(I D, R_{2}\right) \bmod 3, t_{2}$ is hashed to $1 ; t_{3}$ is hashed to $0 ; t_{5}$ is hashed to 2 ; they are hashed to different $b \in[0,2]$ 一this 3 -collision slot is successfully reconciled. Another example: tags $t_{4}$ and $t_{6}$ confined in a 2-collision slot are hashed to the same number $b \in[0,1]$ by performing $H\left(I D, R_{2}\right) \bmod 2$-this 2 collision slot is unreconcilable. The elements corresponding to the $u n$ reconcilable collision slots are re-set to ' $0 \mathrm{~s}$ '.

How to relocate the tags in the reconcilable $k$-collision slot? Obviously, the reader is able to know which $k$ collision slots ( $k=2$ or 3$)$ are reconcilable. For an arbitrary reconcilable $k$-collision slot, let $\lambda_{2}$ denote the number of reconcilable 2-collision slots preceding it; similarly, let $\lambda_{3}$ denote the number of reconcilable 3collision slots preceding it. Clearly, the $k$ tags confined in this reconcilable $k$-collision slot are hashed to different $b \in[0, k-1]$. The tag that is hashed to 0 still stays in this slot; and the tag that is hashed to $b \in[1, k-1]$ is relocated to the $\left[\left(\lambda_{2} \times 1+\lambda_{3} \times 2\right)+b\right]^{t h}$ slot in the appended vector - the appended vector is a vector with dynamic length appended to the tail of the filter vector. As exemplified in Fig. 1 (b), for the first reconcilable 3 -collision slot, $t_{3}$ is hashed to 0 when reconciling, so it still stays in this slot; $t_{2}$ is hashed to 1 , then it is relocated to the $1^{\text {st }}$ slot in the appended vector; $t_{5}$ is hashed to 2 , then it is relocated to the $2^{\text {nd }}$ slot in the appended vector. Another example is given in the following. For the second reconcilable collision slot (a 2-collision slot), $t_{8}$ is hashed to 0 when reconciling, then it still stays in the original slot; $t_{7}$ is hashed to 1 , and because there is one reconcilable 3 collision slot preceding this slot, then it is relocated to the $(1 \times 2+1)^{t h}$ slot (i.e., the $3^{r d}$ slot) in the appended vector.

\subsubsection{Slot Filtering Stage}

Recall that the elements in the filter vector corresponding to the unreconcilable collision slots are reset to ' $0 \mathrm{~s}$ '. As a result, each of the non-zero elements in the filter vector represents either an expected singleton slot or a reconcilable $k$-collision slot $(k=2$ or 3$)$. Let $\bar{\chi}$ denote the number of all non-zero elements in the filter vector. In the next stage, only the slots corresponding to non-zero elements in the filter vector and in the appended vector are executed. The expected empty slots, the $k$-collision slots $(k \geq 4)$ and the unreconcilable $k$-collision slots $(k=2$ or 3$)$ that correspond to ' $0 \mathrm{~s}$ ' in the filter vector are directly skipped, namely, filtered out.

\subsubsection{Presence Verifying Stage}

The reader broadcasts the parameters $R_{1}, R_{2}, f, \bar{\chi}$, and the filter vector to the tags. Each tag, say $t_{i}$, first uses the received parameters and its ID to calculate $s_{i}^{\prime t h}=H\left(I D_{i}, R_{1}\right) \bmod f$. When receiving the filter vector broadcasted by the reader, tag $t_{i}$ records the number of ' $1 \mathrm{~s}^{\prime}$ ', ' $2 \mathrm{~s}$ ', and ' $3 \mathrm{~s}^{\prime}$ preceding the $s_{i}^{\text {th }}$ element in the filter vector, denoted as $\chi_{i 1}, \chi_{i 2}$ and $\chi_{i 3}$, respectively. After receiving the $s_{i}^{\prime}$ th element, it checks the $s_{i}^{\prime}$ th element. Then, it picks a slot to respond based on the following rules: 
1) If the $s_{i}^{\prime}$ th element is ' 1 ', which means that $t_{i}$ selects an expected singleton slot, it will respond in the $\left(\chi_{i 1}+\chi_{i 2}+\chi_{i 3}+1\right)^{t h}$ slot.

2) If the $s_{i}^{\prime \text { th }}$ element is ' 2 ', which means that $t_{i}$ selects a reconcilable 2 -collision slot, then it calculates $H\left(I D_{i}, R_{2}\right) \bmod 2$. (i) If the hashing result of $H\left(I D_{i}, R_{2}\right)$ mod 2 is 0 , it will respond in the $\left(\chi_{i 1}+\chi_{i 2}+\chi_{i 3}+1\right)^{t h}$ slot; (ii) if the hashing result of $H\left(I D_{i}, R_{2}\right) \bmod 2$ is 1 , it will respond in the $\left(\bar{\chi}+\chi_{i 2}+\chi_{i 3} \times 2+1\right)^{t h}$ slot.

3) If the $s_{i}^{\text {'th }}$ element is ' 3 ', which means that $t_{i}$ selects a reconcilable 3 -collision slot, then it calculates $H\left(I D_{i}, R_{2}\right) \bmod 3$. (i) If the hashing result of $H\left(I D_{i}, R_{2}\right) \bmod 3$ is 0 , it will respond in the $\left(\chi_{i 1}+\chi_{i 2}+\chi_{i 3}+1\right)^{t h}$ slot; (ii) if the hashing result of $H\left(I D_{i}, R_{2}\right) \bmod 3$ is 1 , it will respond in the $\left(\bar{\chi}+\chi_{i 2}+\chi_{i 3} \times 2+1\right)^{t h}$ slot; (iii) if the hashing result of $H\left(I D_{i}, R_{2}\right) \bmod 3$ is 2 , it will respond in the $\left(\bar{\chi}+\chi_{i 2}+\chi_{i 3} \times 2+2\right)^{t h}$ slot.

4) If the $s_{i}^{\prime}$ th element is ' 0 ', which means that (i) $t_{i}$ selects an unreconcilable $k$-collision slot $(k=2$ or 3 ); or (ii) $t_{i}$ selects a $k$-collision slot $(k \geq 4)$, which is hard to reconcile and is directly abandoned in this paper. As a result, tag $t_{i}$ will not respond in this round.

Because all the used parameters and the hashing function $H(\cdot)$ are shared by the reader and all the tags, the reader is able to predict all the decisions of tags. Clearly, all the executed slots are expected to be the singleton slots. If the reader does not receive a response in a slot as expected, the tag corresponding to this slot must be missing.

In this round, the presence of the tags that pick the expected singleton slots or reconcilable collision slots can be verified, and they will not participate the following rounds. As illustrated in Fig. 1 (b), all tags excluding $t_{4}$ and $t_{6}$ can be verified. However, tags $t_{4}$ and $t_{6}$ can not be verified because they pick an unreconcilable collision slots. As aforementioned, SFMTI repeats for multiple rounds until the presence of all tags are verified.

Note that the filter vector broadcasted in the third stage may be too long to be transmitted in one tag slot (i.e., $\left.t_{t a g}\right)$. To address this problem, the long filter vector is divided into multiple segments of 96-bits to be sequentially transmitted in multiple tag slots [8].

\subsection{Determining the Optimal Filter Vector Length}

In what follows, we will present how to choose the optimal filter vector length $f$ in order to achieve the best time-efficiency. In an arbitrary round of executing the SFMTI protocol, let $N^{*}$ denote the number of tags that are not verified and will participate in this round.

For a certain element in the filter vector, the corresponding slot can be used only if (1) it is an expected singleton slot; or (2) it is a reconcilable 2-collision slot; or (3) it is a reconcilable 3 -collision slot. Let $P_{1}, P_{2}$,
$P_{3}$ denote the probability that this slot is an expected singleton slot, is a reconcilable 2-collision slot, and is a reconcilable 3 -collision slot, respectively. They are given as follows:

$$
\begin{aligned}
P_{1} & =\left(\begin{array}{c}
N^{*} \\
1
\end{array}\right) \times \frac{1}{f} \times\left(1-\frac{1}{f}\right)^{N^{*}-1} \\
& \approx \frac{N^{*}}{f} \times e^{-\frac{N^{*}-1}{f}} \\
& \approx \rho e^{-\rho}
\end{aligned}
$$

where $\frac{N^{*}}{f}$ is denoted as $\rho$ for the clarity. Note that, because the length $f$ of the filter vector is usually very large, $\left(1-\frac{1}{f}\right)^{N^{*}-1}$ is approximated to $e^{-\frac{N^{*}-1}{f}}$ in Eq. (1).

If this slot is a $k$-collision slot $(k=2$ or 3$)$, the probability that it can be successfully reconciled is $\frac{k !}{k^{k}}$. Then, we have:

$$
\begin{aligned}
P_{2} & =\left[\left(\begin{array}{c}
N^{*} \\
2
\end{array}\right) \times\left(\frac{1}{f}\right)^{2} \times\left(1-\frac{1}{f}\right)^{N^{*}-2}\right] \times \frac{2 !}{2^{2}} \\
& \approx \frac{N^{*}\left(N^{*}-1\right)}{4 f^{2}} \times e^{-\frac{N^{*}-2}{f}} \\
& \approx \frac{1}{4} \rho^{2} e^{-\rho}, \\
P_{3} & =\left[\left(\begin{array}{c}
N^{*} \\
3
\end{array}\right) \times\left(\frac{1}{f}\right)^{3} \times\left(1-\frac{1}{f}\right)^{N^{*}-3}\right] \times \frac{3 !}{3^{3}} \\
& \approx \frac{N^{*}\left(N^{*}-1\right)\left(N^{*}-2\right)}{27 f^{3}} \times e^{-\frac{N^{*}-3}{f}} \\
& \approx \frac{1}{27} \rho^{3} e^{-\rho},
\end{aligned}
$$

There are $f$ elements (i.e., slots) in the filter vector, each having the probability $P_{1}$ to be an expected singleton slot; having the probability $P_{2}$ to be a reconcilable 2-collision slot; and having the probability $P_{3}$ to be a reconcilable 3 -collision slot. Let $\aleph_{1}, \aleph_{2}, \aleph_{3}$ denote the expected number of singleton slots, reconcilable 2collision slot, reconcilable 3-collision slot, respectively. We have:

$$
\begin{aligned}
& \aleph_{1}=f \times P_{1} \approx f \times \rho e^{-\rho} \\
& \aleph_{2}=f \times P_{2} \approx f \times \frac{1}{4} \rho^{2} e^{-\rho} \\
& \aleph_{3}=f \times P_{3} \approx f \times \frac{1}{27} \rho^{3} e^{-\rho}
\end{aligned}
$$

Note that, each reconcilable $k$-collision slot $(k=2$ or 3 ) is reconciled into $k$ singleton slots finally. As a result, $\aleph_{1}+2 \aleph_{2}+3 \aleph_{3}$ expected singleton slots are achieved. We use $T$ to denote the execution time of this round, which includes the time for transmitting the filter vector ( $2 f$ bits) and the $\aleph_{1}+2 \aleph_{2}+3 \aleph_{3}$ shortresponse slots. Hence, $T$ is given as follows:

$$
T=\left\lceil\frac{2 f}{96}\right\rceil \times t_{\text {tag }}+\left(\aleph_{1}+2 \aleph_{2}+3 \aleph_{3}\right) \times t_{\text {short }}
$$

Since each of the $\aleph_{1}+2 \aleph_{2}+3 \aleph_{3}$ singleton slots can be used to verify the presence of a tag, the number of tags that can be verified in this round, denoted as $\Re$, can be given as follows: 


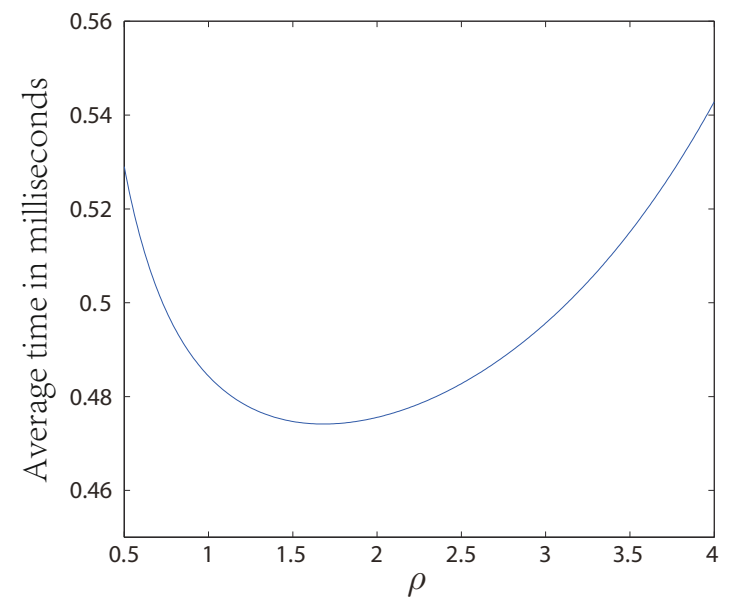

Fig. 2. The average execution time for verifying a tag with respect to $\rho$.

$$
\Re=\aleph_{1}+2 \aleph_{2}+3 \aleph_{3}
$$

According to Eqs. (5) and (6), the average time for verifying the presence of one tag is given as follows:

$$
\begin{aligned}
\frac{T}{\Re} & =\frac{\left\lceil\frac{2 f}{96}\right\rceil \times t_{\text {tag }}+\left(\aleph_{1}+2 \aleph_{2}+3 \aleph_{3}\right) \times t_{\text {short }}}{\aleph_{1}+2 \aleph_{2}+3 \aleph_{3}} \\
& =\frac{\left\lceil\frac{2 f}{96}\right\rceil \times t_{\text {tag }}+\left(f \rho e^{-\rho}+\frac{1}{2} f \rho^{2} e^{-\rho}+\frac{1}{9} f \rho^{3} e^{-\rho}\right) \times t_{\text {short }}}{f \rho e^{-\rho}+\frac{1}{2} f \rho^{2} e^{-\rho}+\frac{1}{9} f \rho^{3} e^{-\rho}} \\
& \approx \frac{\frac{t_{t a g}}{48}+\left(\rho e^{-\rho}+\frac{1}{2} \rho^{2} e^{-\rho}+\frac{1}{9} \rho^{3} e^{-\rho}\right) \times t_{\text {short }}}{\rho e^{-\rho}+\frac{1}{2} \rho^{2} e^{-\rho}+\frac{1}{9} \rho^{3} e^{-\rho}}
\end{aligned}
$$

where $t_{\text {tag }}=2.4 \mathrm{~ms}$ and $t_{\text {short }}=0.4 \mathrm{~ms}$. As illustrated in Fig. 2, the average time spent per tag, $\frac{T}{\Re}$, is a function of $\rho$. To achieve the best time-efficiency, we need to minimize $\frac{T}{\Re}$ in Eq. (7). Hence, we get its derivative as follows:

$$
\left(\frac{T}{\Re}\right)^{\prime}=\frac{-\frac{t_{t a g}}{48} \times e^{-\rho} \times\left(1-\frac{1}{6} \rho^{2}-\frac{1}{9} \rho^{3}\right)}{\left(\rho e^{-\rho}+\frac{1}{2} \rho^{2} e^{-\rho}+\frac{1}{9} \rho^{3} e^{-\rho}\right)^{2}}
$$

It is easy to get a $\rho_{0}$ to satisfy the $\left(\frac{T}{\Re}\right)^{\prime}=0$ in Eq. (8). Clearly, $\left(\frac{T}{\Re}\right)^{\prime}$ is larger than 0 when $\rho>\rho_{0}$; and $\left(\frac{T}{\Re}\right)^{\prime}$ is smaller than 0 when $\rho<\rho_{0}$. That is, $\frac{T}{\Re}$ is minimized when $\rho=\rho_{0}$, where $\rho_{0} \approx 1.68$ and the average execution time per tag, $\frac{T}{\Re}$, is approximately equal to $0.474 \mathrm{~ms}$. Clearly, $\frac{T}{\Re}$ only depends on $\rho$ (i.e., the ratio $\frac{N^{*}}{f}$ ) instead of $N^{*}$. Hence, if we set $\rho=1.68$ (i.e., $f=\frac{N^{*}}{1.68}$ ) for all rounds, the average execution time spent to verify a tag becomes a constant $0.474 \mathrm{~ms}$ across all the rounds of SFMTI. As a result, the execution time of the proposed SFMTI is $0.474 \mathrm{Nms}$, where $N$ is the number of all tags that need to be monitored.

\subsection{Estimating The Expected Execution Round Count $C$}

In the previous subsection, we have investigated the optimal length $f$ of the filter vector in each round. The filter vector length $f$ is optimized to $\frac{N^{*}}{1.68}$ (i.e., $\rho=1.68$ ) in an arbitrary round, where $N^{*}$ is the number of tags that participate in this round. Under this condition, we estimate how many rounds the SFMTI protocol needs to execute to verify the presence of all RFID tags.

Let $\theta$ denote the ratio of the tags that can be verified in an arbitrary round. According to Eqs. (4) and (6), $\theta$ is given as follows:

$$
\begin{aligned}
\theta & =\frac{\Re}{N^{*}}=\frac{\aleph_{1}+2 \aleph_{2}+3 \aleph_{3}}{N^{*}} \\
& =e^{-\rho}\left(1+\frac{1}{2} \rho+\frac{1}{9} \rho^{2}\right),
\end{aligned}
$$

where $\rho=1.68$. That is, the ratio $\theta$ of the tags that can be verified in this round is a constant. The other $1-\theta$ of the $N^{*}$ tags cannot be verified and will participate in the next round. It is easy to know that the number of tags that participate in the $C^{t h}$ round is $N \times(1-$ $\theta)^{C-1}$ (expectation value). Using the knowledge that this value is a positive integer, we get the following inequality:

$$
N \times(1-\theta)^{C-1} \geq 1
$$

By solving Eq. (10), we get $C \leq \log _{(1-\theta)}\left(\frac{1}{N}\right)+1$, where $\theta$ is a constant given in Eq. (9). That is, the execution round count of SFMTI is bounded by $\log _{(1-\theta)}\left(\frac{1}{N}\right)+1$.

\subsection{Extension: Considering Multiple Readers}

In this subsection, we propose an effective method to extend SFMTI for use in the multi-reader scenarios. Considering $\eta$ readers are deployed in a large-scale area, and they are denoted as $r_{i} \mid i \in[1, \eta]$. We assume all the tags are uniformly placed and covered by at least one reader, the tag set covered by reader $r_{i}$ is denoted as $\gamma_{i}$. Obviously, we have $\gamma_{1} \bigcup \gamma_{2} \bigcup, \cdots, \bigcup \gamma_{\eta}=$ $S_{\text {all }}-S_{m i s s}$; and $S_{m i s s}=\emptyset$ when no tag is missing. Note that, if the adjacent readers simultaneously interrogate the overlapped tags, reader-collision will occur. In [24], Yang et al. investigated a protocol stack named Season to solve this reader-collision problem. Briefly, an identification procedure is splitted into two phases. In Phase-I (Shelving Interference), the system identifies all non-contentious tags. In Phase-II (Joint Identification), neighboring readers jointly and collaboratively identify contentious tags. Due to the limitation of space, we do not discuss this issue in detail.

In the multi-reader scenarios, how to let a reader know which tags are confined in its interrogating range is a challenging issue. Fortunately, this can be achieved using the well-known Bloom filter technique [25], [26]. A Bloom Filter is a data structure that probabilistically represents a set of $n$ elements $Y=\left\{y_{1}, y_{2}, \cdots, y_{n}\right\}$, which can be used to test set membership. Specially, each of the $n$ elements in this 
set is compressed into a Bloom filter vector with $w$ bits using $k$ hashing functions $h_{1}, h_{2}, \cdots, h_{k}$. A bit in the vector is set to ' 1 ' if at least one element is hashed to that index in the vector. If we want to check whether a given element $y$ belongs to the set $Y$, we compute $h_{1}(y), h_{2}(y), \cdots, h_{k}(y)$ and assert $y \in S$ if and only if all these $k$ bits are ' $1 \mathrm{~s}$ ' in the vector; otherwise, $y \notin Y$. Yue et al. leveraged the synchronized physical layer transmissions to distributively construct the desired Bloom filter, which represents the tag set in the vicinity of a reader [27]. Using the constructed Bloom filter to test the membership of all the IDs in the database, an arbitrary reader, say $r_{i}$, is expected to learn that $\left|\gamma_{i}\right|+p \times\left(N-\left|\gamma_{i}\right|\right)$ tags are within interrogating range, where $p$ is the false positive probability. We denote this tag set as $\hat{\gamma}_{i}$, and it is available to the reader $r_{i}$. It then executes the SFMTI protocol using $\hat{\gamma}_{i}$ as the input, and returns the missing tag set $S_{i}$. In other words, $\hat{\gamma}_{i}-S_{i}$ is the actual tag set within the range of reader $r_{i}$. It is easy to know that $\bigcup_{i=1}^{\eta}\left(\hat{\gamma}_{i}-S_{i}\right)$ is the set of all present tags in the system. Thus, $S_{\text {all }}-\bigcup_{i=1}^{\eta}\left(\hat{\gamma}_{i}-S_{i}\right)$ is the final set of missing tags.

The execution time of this parallelizing method consists of two parts: (1) the time for constructing the Bloom filter distributively; (2) the time for executing the SFMTI protocol in a parallel mode. For an arbitrary reader $r_{i}$, its execution time for constructing the Bloom filter is $-\frac{\left|\gamma_{i}\right| \times \ln p}{(\ln 2)^{2}} \times t_{b i t} m s$, where $t_{b i t}$ is the time (in milliseconds) for transmitting a bit, according to [27]. And the execution time of SFMTI is about $0.474 \times\left(\left|\gamma_{i}\right|+p \times\left(N-\left|\gamma_{i}\right|\right)\right) m s$. The total execution time for $r_{i}$ is $T_{r_{i}}=-\frac{\left|\gamma_{i}\right| \times \ln p}{(\ln 2)^{2}} \times t_{b i t}+0.474\left(\left|\gamma_{i}\right|+p \times\right.$ $\left.\left(N-\left|\gamma_{i}\right|\right)\right) m s$. We need to optimize the false positive $p$ to minimize this execution time. By setting its derivative $\left(T_{r_{i}}\right)^{\prime}=0$, we get $p=\frac{\left|\gamma_{i}\right| \times t_{b i t}}{0.474 \times(\ln 2)^{2}\left(N-\left|\gamma_{i}\right|\right)}$. Moreover, when $p>\frac{\left|\gamma_{i}\right| \times t_{b i t}}{0.474 \times(\ln 2)^{2}\left(N-\left|\gamma_{i}\right|\right)^{\prime}},\left(T_{r_{i}}\right)^{\prime}>0$; when $p<\frac{\left|\gamma_{i}\right| \times t_{b i t}}{0.474 \times(\ln 2)^{2}\left(N-\left|\gamma_{i}\right|\right)},\left(T_{r_{i}}\right)^{\prime}<0$. Hence, the execution time $T_{r_{i}}$ is minimized when $p$ is configured to $\frac{\left|\gamma_{i}\right| \times t_{b i t}}{0.474 \times(\ln 2)^{2}\left(N-\left|\gamma_{i}\right|\right)}$. And the global execution time is the largest execution time of all readers. Note that, there is a problem that the actual set $\gamma_{i}$ of tags that are covered by reader $r_{i}$ is not available in prior. Fortunately, we do not need to know exactly which tags are within $r_{i}$; we only need its number $\left|\gamma_{i}\right|$, which could be roughly approximated by Coverage Acreage of $r_{i} \times$ Tag Density.

\section{Performance Evaluation}

This section will evaluate the performance of the proposed SFMTI protocol. For fair comparison with the most compromising protocol, we adopt the same settings of simulation parameters as IIP [8]. We consider an error-free communication channel and simulate a single reader in the experiments unless otherwise specified. In the Presence Verifying Stage stage, transmission of each segment (96 bits) of the filter vector takes a tag slot (i.e., $t_{t a g}=2.4 \mathrm{~ms}$ ). And it takes each tag a short-response slot (i.e., $t_{\text {short }}=0.4 \mathrm{~ms}$ ) to relay a 1-bit response to the reader for declaring itself. We run each simulation 1000 times and gather the average experimental results.

\subsection{Validate the Optimal Length $f$ of the Filter Vector}

In this set of simulations, we validate the optimal length $f$ of the filter vector, which is the most important parameter setting in the proposed SFMTI protocol. In Section 4.3, we have proven that the filter length $f$ should be set to $\frac{N^{*}}{1.68}$ in each round (i.e., $\left.\rho=\frac{N^{*}}{f}=1.68\right)$. In our simulations, we vary the parameter $\rho$ from 0.5 to 4 , and record the corresponding average execution time per tag. Fig. 3 shows that the simulation results well match the analytical results.

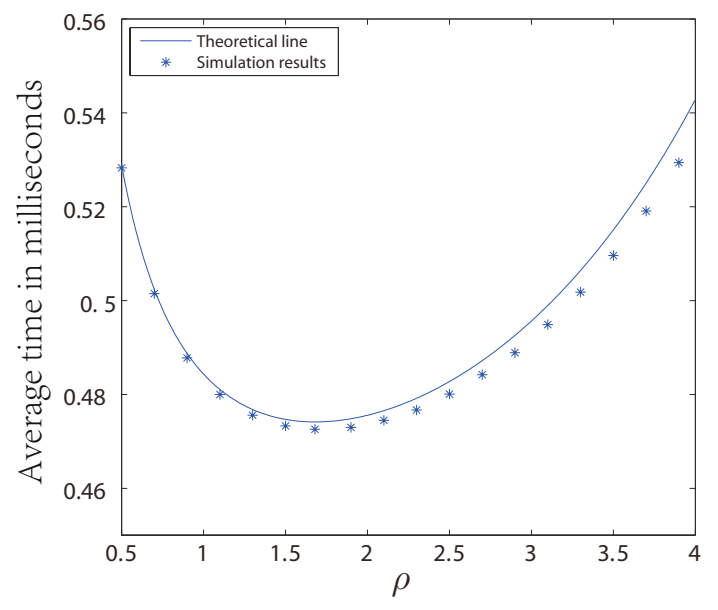

Fig. 3. Fix the number $N$ of all the RFID tags to 10,000 , and vary the parameter $\rho=\frac{N^{*}}{f}$ from 0.5 to 4 , where $N^{*}$ indicates the number of tags that participate in an arbitrary round, and $f$ indicates the length of filter vector in the corresponding round.

\subsection{Validate the Execution Round Count $C$}

In Section 4.4, we have theoretically analyzed the expected execution round count $C$ SFMTI needs to execute so as to verify all RFID tags. Theoretically, the execution round count $C$ is bounded by $\log _{(1-\theta)}\left(\frac{1}{N}\right)+1$, where $N$ is the number of tags and $\theta$ can be obtained according to Eq. (9). In this set of simulations, we validate the expected round count of SFMTI, where we fix the parameter $\rho$ to 1.68 (i.e., the optimal value) and vary the number $N$ of tags from 10,000 to 50, 000 . The simulation results in Table 2 demonstrate that the execution round count (average) of the proposed SFMTI protocol is well bounded by $\log _{(1-\theta)}\left(\frac{1}{N}\right)+1$.

\subsection{Execution Time}

The most important performance criterion for the problem of missing tag identification is the required 
TABLE 3

The execution time with respect to the number $N$ of tags.

\begin{tabular}{|c|c|c|c|c||c|}
\hline \multirow{2}{*}{$\mathrm{N}$} & \multicolumn{5}{|c|}{ Execution time (s) } \\
\cline { 2 - 6 } & BTP & EDFSA & IIP & SFMTI & Lower Bound \\
\hline 5000 & 40.02 & 38.68 & 4.37 & 2.36 & 2.00 \\
\hline 10000 & 80.87 & 77.15 & 8.68 & 4.73 & 4.00 \\
\hline 20000 & 162.04 & 157.23 & 17.29 & 9.47 & 8.00 \\
\hline 30000 & 242.93 & 231.94 & 25.97 & 14.21 & 12.00 \\
\hline 40000 & 324.21 & 311.41 & 34.58 & 18.95 & 16.00 \\
\hline 50000 & 404.46 & 387.80 & 43.21 & 23.69 & 20.00 \\
\hline
\end{tabular}

TABLE 2

Validate the execution round count $C$, with the number $N$ of tags varying from 10,000 to 50,000 .

\begin{tabular}{|c|c|c|c|c|c|}
\hline$N$ & 10,000 & 20,000 & 30,000 & 40,000 & 50,000 \\
\hline Theoretical Round Count & 18.9 & 20.3 & 21.1 & 21.7 & 22.1 \\
\hline Simulation Round Count & 16.5 & 17.9 & 18.6 & 19.2 & 19.7 \\
\hline
\end{tabular}

execution time. We mainly compare the proposed SFMTI protocol with the currently most promising IIP protocol [8]. As the solutions to tag-collection problem can also be used to identify the missing tags, we compare the SFMTI with the well-known tagcollection protocols, including the Enhanced Dynamic Framed Slotted ALOHA (EDFSA) [13] and the Binary Tree Protocol (BTP) [14]. The number of tags, $N$, increases from 5,000 to 50,000 . Table 3 lists the execution time required by these protocols, respectively. The simulation results reveal that the proposed SFMTI protocol performs much better than BTP and EDFSA. For example, when $N=50,000$, the execution time of the BTP and EDFSA is 404.46 and 387.80 seconds, respectively. However, the execution time of the SFMTI protocol is just 23.69 seconds, representing $94.1 \%$ and $93.9 \%$ reduction when compared with the BTP and EDFSA. In comparison with IIP, the SFMTI protocol reduces the execution time by about $45 \%$. For example, When $N$ is 50,000 , the execution time of the SFMTI protocol is $45.2 \%$ less than the time required by the IIP protocol. Moreover, the execution time of the proposed SFMTI protocol is very close to the lower bound of the minimum execution time. For example, when $N=50,000$, the execution time of the SFMTI protocol is 23.69 seconds which is just 1.18 times the lower bound.

\subsection{The Impact of Missing Tag Number}

In this set of simulations, we investigate the impact of the number of missing tags. Besides the total execution time for identifying all the missing tags, the time required for identifying the first missing tag is also an important performance criterion. For example, a thief steals multiple items from a warehouse. Clearly, the faster the protocol identifies the first missing tags, the sooner the alarm is given. We set $N=10,000$ and vary the number of missing tags, $M$, from 10 to 100 . The proposed SFMTI protocol has to verify the presence of all RFID tags so as to completely identify all the missing tags. As illustrated in Fig. 4, with $M$ (the number of missing tags) increasing, the total execution time for identifying all the missing tags keeps unchanged. Whereas, the time for detecting the first missing RFID tag decreases with $M$ increasing, because of an intuitive reason-the more RFID tags are missing, the more easier to detect one of them.

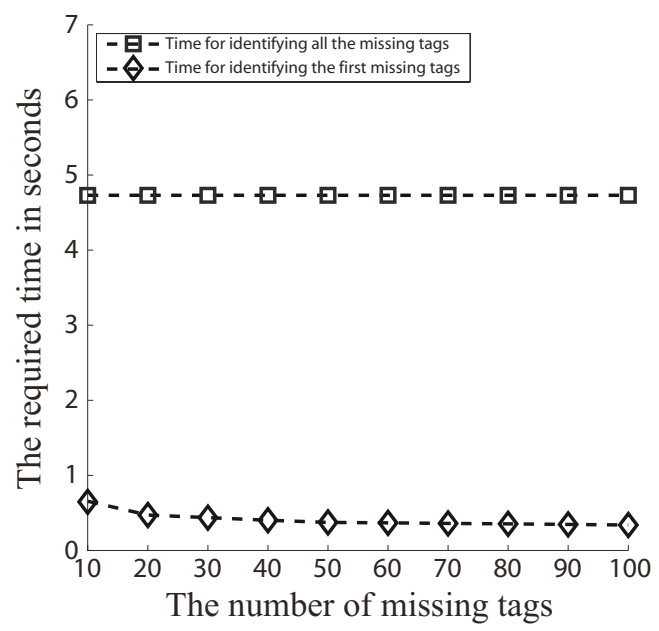

Fig. 4. Fix the number $N$ of the total tags to 10,000 , and vary the number $M$ of the missing tags from 10 to 100.

\subsection{Evaluate the Performance of SFMTI in the Multi-reader Scenarios}

To fully evaluate the performance of the proposed SFMTI protocol, we conducted simulation experiments contain multiple readers in this subsection. As aforementioned, Protocol 3 in [21] performs better than IIP in the multi-reader scenarios. Hence, in this set of simulations, we mainly compare the proposed SFMTI protocol with Protocol 3 in [21]. Following the simulation settings in [21], we simulated a region consisting of square zones, where we use $L$ to denote the number of the readers. The tags are uniformly distributed and $D$ is used to denote the tag density in 
each zone, for example, $D=1000$ tags/zone means that there are 1000 tags in each zone on average. The simulation results in Fig. 5 demonstrate that the proposed SFMTI protocol still performs much better than both IIP and Protocol 3, where the number $L$ of the readers is fixed to 50 and the tag density $D$ varies from 400 tags/zone to 2000 tags/zone. For example, when $D=2000$ tags/zone, $L=50$ (meaning $2000 \times 50=100,000$ tags), the execution time of IIP and Protocol 3 is $86.07 \mathrm{~s}$ and $18.69 \mathrm{~s}$, respectively. And the execution time of SFMTI is just $6.71 \mathrm{~s}$, representing $92.2 \%$ and $64.1 \%$ reduction compared with IIP [8] and Protocol 3 [21].

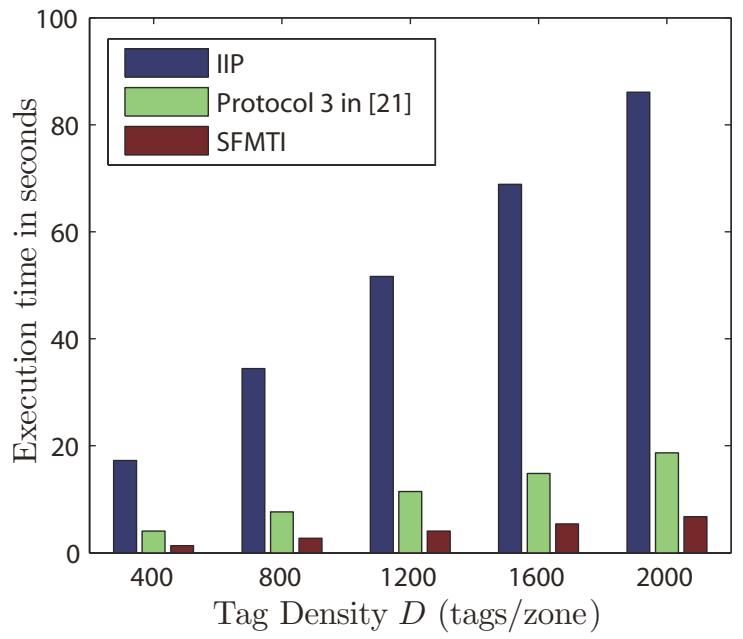

Fig. 5. The number $L$ of the readers is fixed to 50 and the tag density $D$ varies from 400 tags/zone to 2000 tags/zone.

\section{CONCLUSION}

This study has addressed the important problem of complete identification of missing tags in large-scale RFID systems. The solutions to this problem are desirable in many valuable monitoring applications in warehouses, hospitals, prisons, etc. This paper has proposed a Slot Filter-based Missing Tag Identification (SFMTI) protocol to identify the missing tags in a time-efficient way. We have also presented how to choose the optimal parameters of the SFMTI protocol. To accommodate multiple readers deployed in some large area scenarios, we have proposed a cost-effective extension method, in which all the readers work in a parallel mode. Furthermore, extensive simulation experiments have been conducted to evaluate the performance of the SFMTI protocol. The simulation results show that the proposed SFMTI protocol outperforms the currently most promising protocol by reducing $45 \%$ of the required execution time. Moreover, the execution time of the SFMTI protocol is just within a factor of 1.18 from the lower bound of the minimum execution time.

\section{ACKNOWLEDGMENTS}

This work was supported by NSFC (grant nos 61173162, 61173160, 61103234, 61272417, 61173161, 61300189 , and 61173165), the National Science Foundation for Distinguished Young Scholars of China (grant no 61225010), the Fundamental Research Funds for the Central Universities, and Shanghai Key Laboratory of Scalable Computing and Systems.

\section{References}

[1] C. Wang, H. Wu, and N.-F. Tzeng, "RFID-Based 3-D Positioning Schemes," Proc. of IEEE INFOCOM, 2007.

[2] L. M. Ni, Y. Liu, Y. C. Lau, and A. P. Patil, "Landmarc: Indoor Location Sensing Using Active RFID," Wireless Networks, vol. 10, no. 6, pp. 701-710, 2004.

[3] A. Nemmaluri, M. D. Corner, and P. Shenoy, "Sherlock: Automatically Locating Objects for Humans," Proc. of ACM MobiSys, 2008.

[4] B. Sheng, C. C. Tan, Q. Li, and W. Mao, "Finding Popular Categories for RFID Tags," Proc. of ACM MobiHoc, 2008.

[5] C. Qian, H. Ngan, Y. Liu, and L. M. Ni, "Cardinality Estimation for Large-Scale RFID Systems," IEEE Transactions on Parallel and Distributed Systems, vol. 22, no. 9, pp. 1441-1454, 2011.

[6] C. Lee and C. Chung, "Efficient Storage Scheme and Query Processing for Supply Chain Management using RFID," Proc. of ACM SIGMOD, 2008

[7] M. Kodialam, T. Nandagopal, and W. C. Lau, "Anonymous Tracking using RFID tags," Proc. of IEEE INFOCOM, 2007.

[8] T. Li, S. Chen, and Y. Ling, "Identifying the Missing Tags in a Large RFID System," Proc. of ACM MobiHoc, 2010.

[9] Y. Zheng and M. Li, "Fast Tag Searching Protocol for LargeScale RFID Systems," Proc. of IEEE ICNP, 2011

[10] M. Shahzad and A. X. Liu, "Every Bit Counts-Fast and Scalable RFID Estimation," Proc. of ACM MobiCom, 2012.

[11] F. C. Schoute, "Dynamic Frame Length ALOHA," IEEE Transactions on Communications, vol. 31, no. 4, pp. 565 - 568, 1983.

[12] L. G. Roberts, "Aloha Packet System with and without Slots and capture," ACM SIGCOMM Computer Communication Review, vol. 5, no. 2, pp. 28-42, 1975.

[13] S. Lee, S. Joo, and C. Lee, "An Enhanced Dynamic Framed Slotted ALOHA Algorithm for RFID Tag Identification," Proc. of IEEE MobiQuitous, 2005.

[14] J. Myung and W. Lee, "Adaptive Splitting Protocols for RFID Tag Collision Arbitration," Proc. of ACM MobiHoc, 2006.

[15] N. Bhandari, A. Sahoo, and S. Iyer, "Intelligent Query Tree (IQT) Protocol to Improve RFID Tag Read Efficiency," Proc. of IEEE ICIT, 2006.

[16] V. Namboodiri and L. Gao, "Energy-Aware Tag Anticollision Protocols for RFID Systems," IEEE Transactions on Mobile Computing, vol. 9, no. 1, pp. 44-59, 2010.

[17] M. Shahzad and A. X. Liu, "Probabilistic Optimal Tree Hopping for RFID Identification," Proc. of ACM SIGMETRICS, 2013

[18] C. C. Tan, B. Sheng, and Q. Li, "Efficient Techniques for Monitoring Missing RFID Tags," IEEE Transactions on Wireless Communications, vol. 9, no. 6, pp. 1882-1889, 2010.

[19] W. Luo, S. Chen, T. Li, and S. Chen, "Efficient Missing Tag Detection in RFID Systems," Proc. of IEEE INFOCOM, 2011.

[20] W. Luo, S. Chen, T. Li, and Y. Qian, "Probabilistic Missingtag Detection and Energy-Time Tradeoff in Large-scale RFID systems," Proc. of ACM MobiHoc, 2012.

[21] R. Zhang, Y. Liu, Y. Zhang, and J. Sun, "Fast Identification of the Missing Tags in a Large RFID System," Proc. of IEEE SECON, 2011.

[22] H. Han, B. Sheng, C. C. Tan, Q. Li, W. Mao, and S. Lu, "Counting RFID Tags Efficiently and Anonymously," Proc. of IEEE INFOCOM, 2010

[23] P. Semiconductors, "I-CODE Smart Label RFID Tags," http://www.nxp.com/acrobat_download/other/identification/ SL092030.pdf, Jan 2004

[24] L. Yang, J. Han, C. Wang, T. Gu, and Y. Liu, "Season: Shelving Interference and Joint Identification in Large-scale RFID Systems," Proc. of IEEE INFOCOM, 2011. 
[25] B. Bloom, "Space/time tradeoffs in hash coding with allowable errors," Communications of the ACM, vol. 13, no. 7, pp. 422-426.

[26] H. Fang, K. Murali, and L. TV, "Building high accuracy bloom filters using partitioned hashing," vol. 35, no. 1, pp. 277-288, 2007.

[27] H. Yue, C. Zhang, M. Pan, Y. Fang, and S. Chen, "A Timeefficient Information Collection Protocol for Large-scale RFID Systems," Proc. of IEEE INFOCOM, 2012.

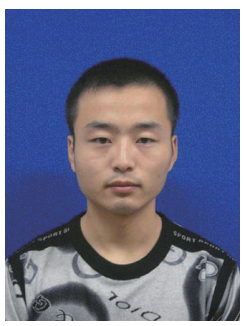

Xiulong Liu received the B.E. degree from the School of Software Technology, Dalian University of Technology, China, in 2010. Currently, he is a Ph.D. candidate in the School of Computer Science and Technology, Dalian University of Technology, China. His research interests include RFID systems and wireless sensor networks.

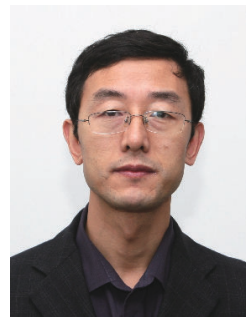

Keqiu Li received the bachelor's and master's degrees from the Department of Applied Mathematics at the Dalian University of Technology in 1994 and 1997, respectively. $\mathrm{He}$ received the Ph.D. degree from the Graduate School of Information Science, Japan Advanced Institute of Science and Technology in 2005. He also has two-year postdoctoral experience in the University of Tokyo, Japan. $\mathrm{He}$ is currently a professor in the School of Computer Science and Technology, Dalian University of Technology, China. He has published more than 100 technical papers, such as IEEE TPDS, ACM TOIT, and ACM TOMCCAP. $\mathrm{He}$ is an Associate Editor of IEEE TPDS and IEEE TC. He is a senior member of IEEE. His research interests include internet technology, data center networks, cloud computing and wireless networks.

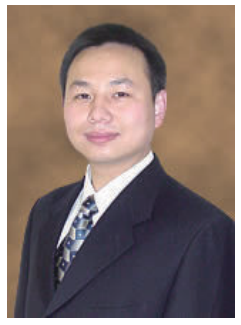

Geyong Min is a Professor of Computer Science in the Department of Computing at the University of Bradford, United Kingdom. $\mathrm{He}$ received the $\mathrm{PhD}$ degree in Computing Science from the University of Glasgow, United Kingdom, in 2003, and the B.Sc. degree in Computer Science from Huazhong University of Science and Technology, China, in 1995. His research interests include Next Generation Internet, Wireless Communications, Multimedia Systems, Information Security, Ubiquitous Computing, Modelling and Performance Engineering. His recent research has been supported by UK EPSRC, Royal Society, Nuffield Foundation, and European FP. Prof. Min has published over 200 research papers in prestigious international journals, including IEEE Transactions on Computers, IEEE Transactions on Parallel and Distributed Systems, IEEE Transactions on Communications, IEEE Transactions on Wireless Communications, and IEEE Transactions on Multimedia, and in reputable international conferences, such as ICDCS, IPDPS, GLOBECOM, and ICC. He is an Editorial Board member of 9 international journals. He served as the Guest Editor for 17 International Journals and was the Chair or Vice-Chair of 30 international conferences/workshops. He was awarded the Outstanding Leadership Awards from IEEE International conferences CIT'2010/ScalCom'2010/ICESS'2010, ScalCom'2009, HPCC'2008.

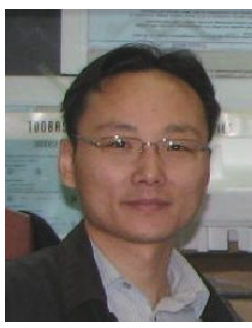

Yanming Shen received the B.S degree in automation from Tsinghua University, Beijing, China, in 2000, and the Ph.D. degree from the Department of Electrical and Computer Engineering at the Polytechnic Institute of New York University, Brooklyn, in 2007. $\mathrm{He}$ is a Professor with the School of Computer Science and Technology, Dalian University of Technology, DaLian, China. His general research interests include packet switch design, data center networks, peer-topeer video streaming, and algorithm design, analysis, and optimization. He is a recipient of the 2011 Best Paper Awards for Multimedia Communications (awarded by IEEE Communications Society).

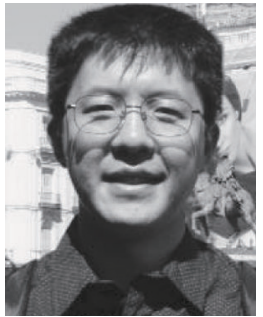

Alex X. Liu received his Ph.D. degree in computer science from the University of Texas at Austin in 2006. He is an Associate Professor with the Department of Computer Science and Engineering, Michigan State University. $\mathrm{He}$ is an Associate Editor of IEEE/ACM Transactions on Networking and an Area Editor of Elsevier Journal of Computer Communications. He received the IEEE \& IFIP William C. Carter Award in 2004 and an NSF CAREER award in 2009. He received the Withrow Distinguished Scholar Award in 2011 at Michigan State University. He received Best Paper Awards from ICNP2012, SRDS-2012, and LISA-2010. His research interests focus on networking and security.

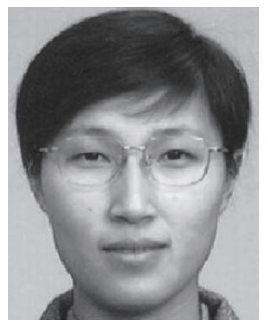

Weyu Qu received the bachelor's and master's degrees from Dalian University of Technology, China in 1994 and 1997, and the doctorate degree from Japan Advanced Institute of Science and Technology in 2006. She is a professor at the School of Information and Technology, Dalian Maritime University, China. She was a lecturer in Dalian University of Technology from 1997 to 2003 . Her research interests include mobile agent-based technology, distributed computing, computer networks, and grid computing. She has published more than 50 technical papers in international journals and conferences. She is on the committee board for a couple of international conferences. 